\section{Ocular Emergencies in Disasters}

Dr. David Mallek, $M D$

Vancouver, British Columbia, CANADA

When large groups of people are in fires, explosions, earthquakes, or wars, there can occur many ocular injuries. These can be contaminated, sterile, or chemical. The patients may not be aware of the injury or may be unconscious. Treatment begins in the field with sterile, nonpressure dressings, incident labeling, and early evacuation. Charting starts at triage with careful attention to patient identification for tracking and pictorial descriptions. Every person should be checked quickly for trauma.

Surgical care is easier with portable microscopes as eye care can follow neurosurgical or orthopedic procedures in the same room and with the same anesthetic. Roving ophthalmic follow-up assures continuity despite other treatments requiring that the patient be moved all around the hospital.

Keywords: eye; injuries; ophthalmology; surgery; trauma
Taiwan's Problems of Disaster Management from the Viewpoint of a 500-bed Hospital Hit Hard by an Earthquake

Dr. Chan-Liao Mingi

Department of Anesthesia, Jen Ai Hospital, Tali, Taichung, Taiwan ROC

A devastating earthquake measured 7.6 (Richter scale) struck Central Taiwan on 21 September 1999, and was responsible for mass casualties. The official data indicated that there were more than 2,400 fatalities, 10,000 injuries, and a cost of US $\$ 10$ billion. A total of 468 health-careproviding units reported to absorb the various degrees of building damage of which 87 suffered collapse; 115 major damage, and the remaining, minor damage. Our 500-bed hospital was one of the few big hospitals severely damaged by the earthquake. We report our experience at managing and operating in a rather chaotic disaster situation.

A recent opinion poll indicated that $>50 \%$ of the residents from the impact areas were dissatisfied with government's response to the disaster. So in order to analyze the problems associated with the relief efforts and to determine the societal impacts, we also investigated the existing literature, journalistic accounts, and official documents concerning the earthquake.

We expect relief efforts by local citizens and relevant county and city governments as well as national agencies will be improved and better coordinated with time and improved technology. Taiwan still is rebuilding following this deadly earthquake. At this time, a refined protocol and a nationwide disaster plan are being developed.

Keywords: collapse, building; disaster; earthquake; hospitals; opinion, public; relief 